\title{
Outcome of postoperative radiotherapy following radical prostatectomy: a single institutional experience
}

\author{
Sea-Won Lee, MD', Tae-Kon Hwang, MD², Sung-Hoo Hong, MD², Ji-Youl Lee, MD², \\ Mi Joo Chung, MD', Song Mi Jeong, MD', Sung Hwan Kim, MD', \\ Jong Hoon Lee, MD', Hong Seok Jang, $\mathrm{MD}^{3}$, Sei Chul Yoon, $\mathrm{MD}^{4}$ \\ 'Department of Radiation Oncology, St. Vincent's Hospital, The Catholic University of Korea College of Medicine, Suwon; \\ Departments of ${ }^{2}$ Urology and ${ }^{3}$ Radiation Oncology, Seoul St. Mary's Hospital, The Catholic University of Korea \\ College of Medicine, Seoul; ${ }^{4}$ Department of Radiation Oncology, Bucheon St. Mary's Hospital, \\ The Catholic University of Korea College of Medicine, Bucheon, Korea
}

Purpose: This single institutional study is aimed to observe the outcome of patients who received postoperative radiotherapy after radical prostatectomy.

Materials and Methods: A total of 59 men with histologically identified prostate adenocarcinoma who had received postoperative radiation after radical prostatectomy from August 2005 to July 2011 in Seoul St. Mary's Hospital of the Catholic University of Korea, was included. They received 45-50 Gy to the pelvis and boost on the prostate bed was given up to total dose of 63-72 Gy (median, 64.8 Gy) in conventional fractionation. The proportion of patients given hormonal therapy and the pattern in which it was given were analyzed. Primary endpoint was biochemical relapse-free survival (bRFS) after radiotherapy completion. Secondary endpoint was overall survival (OS). Biochemical relapse was defined as a prostate-specific antigen level above $0.2 \mathrm{ng} / \mathrm{mL}$. Results: After median follow-up of 53 months (range, 0 to 104 months), the 5-year bRFS of all patients was estimated $80.4 \%$. The 5 -year OS was estimated 96.6\%. Patients who were given androgen deprivation therapy had a 5 -year bRFS of 95.1\% while the ones who were not given any had that of $40.0 \%(p<0.01)$. However, the statistical significance in survival difference did not persist in multivariate analysis. The 3 -year actuarial grade 3 chronic toxicity was $1.7 \%$ and no grade 3 acute toxicity was observed.

Conclusion: The biochemical and toxicity outcome of post-radical prostatectomy radiotherapy in our institution is favorable and comparable to those of other studies.

Keywords: Prostatic neoplasmsr, Radiation, Prostatectomy, Androgen deprivation therapy

\section{Introduction}

Prostate cancer is one of the most common cancers of men in the Western Hemisphere. The incidence of prostate malignancy is rapidly rising in Asia along with the globalization of lifestyle. The data presented by the Korea Central Cancer Registry in
2010 rated prostate cancer as the fifth leading cause of newly diagnosed cancers in South Korean men [1].

Cancers of the prostate is known to be treated with multidisciplinary approach of surgery, radiation, and androgen deprivation therapy (ADT). Adjuvant therapy is indicated when radical prostatectomy specimen shows adverse pathologic

Received 25 April 2014, Revised 2 July 2014, Accepted 4 July 2014.

Correspondence: Jong Hoon Lee, MD, Department of Radiation Oncology, St. Vincent's Hospital, 93 Jungbu-daero, Paldalgu, Suwon 442-723, Korea. Tel: +82-31-249-7560, Fax: +82-31-257-3734, E-mail: koppul@catholic.ac.kr

(c) This is an Open Access article distributed under the terms of the Creative Commons Attribution Non-Commercial License (http://creativecommons.org/ licenses/by-nc/3.0/) which permits unrestricted non-commercial use, distribution, and reproduction in any medium, provided the original work is properly cited.

www.e-roj.org 
features predictive of recurrence, such as extracapsular extension or positive surgical margin [2]. In such cases with pathologic risk factors, radiation given after radical prostatectomy have produced better outcome [3-7].

By the 1980's new hormonal agents with improved cardiotoxicity were developed and many studies investigating the effect of androgen suppression in addition to radiotherapy (RT) commenced [8]. RTOG 86-10 and EORTC 22961 were the two studies devised to test the role of ADT given short-term in addition to RT [9-11]. In the studies mentioned above the combined modality arms achieved better outcome.

In light of these findings a retrospective review was designed to observe the biochemical and toxicity outcome of our patients who received postoperative RT after radical prostatectomy in Seoul St. Mary's Hospital of the Catholic University of Korea. Although the standard of care for prostate cancer is elucidated to some degree through numerous randomized trials, yet myriad questions remain to be answered as to the sequence, timing and combination of surgery, radiation and $A D T$. This single institutional experience may provide with further information to refer to in making clinical judgments for prostate cancer patients who have received radical prostatectomy.

\section{Materials and Methods}

\section{Patients}

We enrolled a total of 59 men with prostate cancer who have received postoperative RT from August 2005 to July 2011 in Seoul St. Mary's Hospital. The inclusion criteria were histologically proven adenocarcinoma of the prostate status post radical prostatectomy with or without pelvic lymph node dissection. Pelvic lymph node dissection was performed in patients who had Gleason scores more than 7, prostatespecific antigen (PSA) level above 10 or equivocal clinical nodal staging in need of surgical staging. Patients with risks of ureteral and neurovascular complication in perioperative setting were omitted the procedure.

Sixty-one percent of the patients received adjuvant radiation therapy, which was given after median 1.3 month after radical prostatectomy. Thirty-nine percent of the patients received salvage radiation therapy given after three successive PSA rises after reaching the PSA nadir achieved by surgery. Patients with distant metastatic disease at the time of RT or the ones given radiation for palliative aim, or men with PSA $\geq 100 \mathrm{ng} / \mathrm{mL}$ were deemed ineligible. All procedures required to perform this study were reviewed by the Institutional Review Board.

The included patients were classified within the risk groups, from low to very high, described in the National Cancer Center Network Guidelines. All surgical pathology were thoroughly reviewed, including pathologic $\mathrm{T}$ and $\mathrm{N}$ staging according to the 2009 American Joint Committee on Cancer Staging System (7th edition), both the major and minor Gleason scores, extracapsular extension, seminal vesicle invasion, resection margin, prostatic urethral invasion, lymphovascular invasion, and perineural invasion. Whether the subjects were treated with androgen depressive agents had been analyzed as well. The patients were followed up every 3 to 6 months for the first 5 years after radical prostatectomy. They annually visited urology clinic since then. Regular follow-up post radiation was done after 1 week, 1 month, 3 months, 6 months, and then yearly thereafter.

\section{Pretreatment evaluation}

Pretreatment evaluation consisted of physical examination including digital rectal exam, serum analysis of complete blood count, blood chemistry, and PSA. PSA levels were acquired initially at diagnosis, before surgery, prior to RT, and at every follow-up. Radiographic assessment were done using chest $X$-ray, KUB, either abdominopelvic computed tomography (CT) or prostate magnetic resonance imaging, and bone scan or positron emission tomography/computed tomography. All patients underwent transrectal ultrasonographic prostate biopsy to confirm malignancy before surgery. Performance status was rated using the Eastern Cooperative Oncology Group (ECOG) scale of which the patients scored from 0 to 2.

\section{Radiotherapy}

Contrast-enhanced CT simulation was performed for all patients lying down on supine position with hands on the chest and both feet stabilized on foot device. Vacuumed cushion covering the entire body was used for intensitymodulated radiation therapy (IMRT) patients. Bladder filling was routinely done both for simulation as well as for each treatment in order to spare the small bowel. Delayed image was taken to define the membranous urethra.

The patients were given 45-50 Gy to the pelvis in four-box fields which covered the pelvic lymphatics superiorly to the lumbosacral junction, inferiorly defined by the lower border of obturator foramen, and $1.5-2 \mathrm{~cm}$ lateral to the pelvic rim. The anterior borders of lateral portals were placed at the anterior aspect of pubic symphysis and the posterior borders were at 
the S2-3 interspace.

The clinical target volume (CTV) for pelvic Iymphatics was contoured with 7-mm margin including both common iliac, external and internal iliac vessels. The obturator lymphatics were drawn above the level of symphysis pubis. The prostate CTV was defined as follows: inferiorly $1 \mathrm{~cm}$ below vesicourethral anastomosis, anteriorly delineated by the posterior border of symphysis pubis until just above the symphysis including bladder neck, posteriorly limited by the anterior rectal wall, and laterally defined by the medial aspect of obturator internus muscles. Above symphysis pubis, the posterosuperior border continued up to include any surgical clip and bilateral neurovascular bundles [12].

The pelvic radiation dose was prescribed $45 \mathrm{~Gy}$ in node negative patients and a higher dose of 50 Gy was given in cases of nodal involvement. The field was then coned down to prostate bed up to total dose of 63-72 Gy (median, 64.8 Gy). Variable margins of $3 \mathrm{~mm}$ to $1 \mathrm{~cm}$ was given to the prostate bed for planning target volume expansion. A daily dose of 1.8-2 Gy was given 5 days a week using a modality of either $3 \mathrm{D}$ conformal radiation therapy (3D-CRT) or IMRT. Most of the patients received $3 \mathrm{D}-\mathrm{CRT}$ considering the cost-effectiveness. A portion of patients who wished IMRT were treated with the latter.

Both the 3D-CRT and IMRT plans were calculated based on the convolution-superposition algorithm. Image guidance was done at the start of treatment with every new plan using electronic portal imaging device for 3D-CRT patients. Daily registration using megavoltage computed tomography (MVCT) was done for IMRT patients. The delivery quality assurance (QA) for IMRT was done using ion chamber array and phantom. The dose distribution of the plan and the measured dose distribution were compared by gamma evaluation of 3 $\mathrm{mm} / 3 \%$.

\section{Androgen deprivation therapy}

ADT was given in patients with rising PSA or with high risk of recurrence, such as positive node in surgical pathology or T3-4 diseases. Combined androgen blockade consisted of leuprorelin intravenous injection (3.75 mg) every month and a tablet of bicalutamide $50 \mathrm{mg}$ orally every day. LHRH agonist regimen was $11.25 \mathrm{mg}$ of leuprorelin intravenously every 3 months. Antiandrogen was given as 1 tablet of $50 \mathrm{mg}$ bicalutamide each day. The duration of hormonal therapy given were divided into $<6,6-12,13-18$, and $>18$ months. The decision to start androgen deprivation was made by the individual urologist's clinical judgment.

\section{Toxicity evaluation}

The toxicities consequent to RT were scored using the Common Terminology Criteria for Adverse Events (CTCAE) v4.0. Among the baseline complications developed after radical prostatectomy, only the aggravating symptoms after RT initiation were regarded as RT-induced toxicities. Acute events were any complication occurring within 90 days of finishing RT. Any side effect developed after that point was considered a late effect. Acute and late effects were subdivided into gastrointestinal, urinary, and genital toxicities.

\section{Endpoint}

Primary endpoint was the biochemical relapse-free survival (bRFS) after the completion of radiation therapy, which was defined as the date of first PSA level increment above 0.2 $\mathrm{ng} / \mathrm{mL}$ after reaching the PSA nadir regardless of clinical progression or death [13]. Secondary endpoint was overall survival (OS). Both bRFS and OS were measured from the date of RT finish.

\section{Statistical analysis}

The survival rates of 5-year bRFS and 5-year OS were estimated using the Kaplan-Meier method. The log-rank test was used to evaluate the significant difference in 5-year bRFS between the two sides in each risk factor subgroup. Multivariate analysis was done by utilizing the Cox proportional hazard models. Statistical significance was evaluated at the 0.05 alpha level. All the tests were two-sided.

\section{Results}

\section{Patients}

The patients were followed-up to a median period of 53 months (range, 0 to 104). One patient expired with the last 2 fractions of his treatment remaining. The cause of death was an upper respiratory tract infection aggravated to sepsis. The patients' characteristics before starting RT are presented in Table 1. Pelvic lymph node dissection was performed in 53\% of patients. Pre-RT PSA for adjuvant cases were median 0.03 (range, 0.004 to 3.15 ) and for salvage cases were median 0.2 (range, 0.01 to 18.93 ).

Adjuvant RT was given median 1.3 months after radical prostatectomy. Salvage RT was given after a median of 16.2 months after the surgery. Radiation therapy was given up to 
Table 1. Patient characteristics

\begin{tabular}{|c|c|c|c|}
\hline & Adjuvant $(n=36)$ & Salvage $(n=23)$ & Total $(n=59)$ \\
\hline Age $(\mathrm{yr})$ & 66 (49-74) & $71.5(56-76)$ & $68(48-76)$ \\
\hline$\leq 65$ & 16 (44.4) & $6(26.1)$ & $22(37.3)$ \\
\hline$>65$ & $20(55.6)$ & 17 (73.9) & $37(62.7)$ \\
\hline \multicolumn{4}{|l|}{ ECOG } \\
\hline 0 & 14 (38.9) & $13(56.5)$ & 27 (45.8) \\
\hline 1 & $22(61.1)$ & $9(39.1)$ & $31(52.5)$ \\
\hline 2 & $0(0)$ & $1(4.3)$ & $1(1.7)$ \\
\hline \multicolumn{4}{|l|}{ Risk group } \\
\hline Very high & $7(19.4)$ & $4(17.4)$ & 11 (18.6) \\
\hline High & $23(63.9)$ & 11 (47.8) & $34(57.6)$ \\
\hline Intermediate & $6(16.7)$ & $6(26.1)$ & $12(20.3)$ \\
\hline Low & $0(0)$ & $2(8.7)$ & $2(3.4)$ \\
\hline Median pre-RT PSA & $0.635(0.004-3.15)$ & $0.2(0.004-18.93)$ & $0.13(0.004-18.93)$ \\
\hline$\leq 0.2$ & 27 (75.0) & $10(43.5)$ & 37 (62.7) \\
\hline$>0.2$ & $9(25.0)$ & $13(56.5)$ & $22(37.3)$ \\
\hline \multicolumn{4}{|l|}{ pT stage } \\
\hline pT2 & $8(22.2)$ & $12(52.2)$ & $20(33.9)$ \\
\hline pT3 & $27(75.0)$ & 11 (47.8) & $38(64.4)$ \\
\hline pT4 & $1(2.8)$ & $0(0)$ & $1(1.7)$ \\
\hline \multicolumn{4}{|l|}{$\mathrm{pN}$ stage } \\
\hline pNO & $20(55.6)$ & $8(34.8)$ & $28(47.5)$ \\
\hline $\mathrm{pN}_{+}$ & $1(2.8)$ & $2(8.7)$ & $3(5.1)$ \\
\hline $\mathrm{pNx}$ & $15(41.7)$ & $13(56.5)$ & $28(47.5)$ \\
\hline \multicolumn{4}{|l|}{ Gleason score } \\
\hline$\leq 6$ & $8(22.2)$ & $5(21.7)$ & $13(22.0)$ \\
\hline 7 & $23(63.9)$ & $13(56.5)$ & $36(61.0)$ \\
\hline $8-10$ & $5(13.9)$ & $5(21.7)$ & $10(16.9)$ \\
\hline \multicolumn{4}{|l|}{ ECE } \\
\hline$(-)$ & $10(27.8)$ & $13(56.5)$ & $23(39.0)$ \\
\hline$(+)$ & $26(72.2)$ & $10(43.5)$ & $36(61.0)$ \\
\hline \multicolumn{4}{|l|}{ SVI } \\
\hline$(-)$ & 30 (83.3) & 19 (82.6) & 49 (83.1) \\
\hline$(+)$ & $6(16.7)$ & 4 (17.4) & $10(16.9)$ \\
\hline \multicolumn{4}{|l|}{ RM } \\
\hline$(-)$ & $4(11.1)$ & $9(39.1)$ & $13(22.0)$ \\
\hline$(+)$ & 32 (88.9) & $14(60.9)$ & $46(78.0)$ \\
\hline \multicolumn{4}{|l|}{ ADT } \\
\hline No & $23(63.9)$ & $13(56.5)$ & 44 (74.6) \\
\hline Yes & 13 (36.1) & $10(43.5)$ & $15(25.4)$ \\
\hline
\end{tabular}

Values are presented as median (range) or number (\%).

ECOG, Eastern Cooperative Oncology Group performance status; RT, radiotherapy; PSA, prostate-specific antigen; ECE, extracapsular extension; SVI, seminal vesicle invasion; RM, resection margin; ADT, androgen deprivation therapy.

median total dose of 6,480 cGy (range, 3,060 to 7,200 cGy) over median duration of 50 days (range, 27 to 64 days). Fiftysix patients (94.9\%) completed RT. One patient developed proctitis and stopped visiting for RT afterwards. Another patient refused RT because the incontinence which first occurred after prostatectomy worsened during radiation. One patient could not finish the planned radiation dose because he expired due to sepsis progressed from an upper respiratory infection.

Approximately a quarter of the patients (25.4\%) were given ADT. Among the 15 patients given hormonal therapy, 12 received combined androgen blockade with the dose described 
A

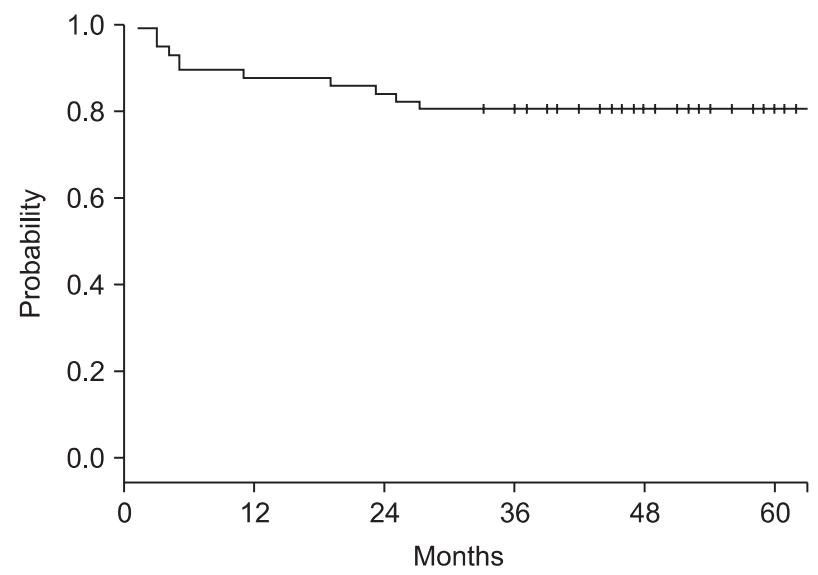

B

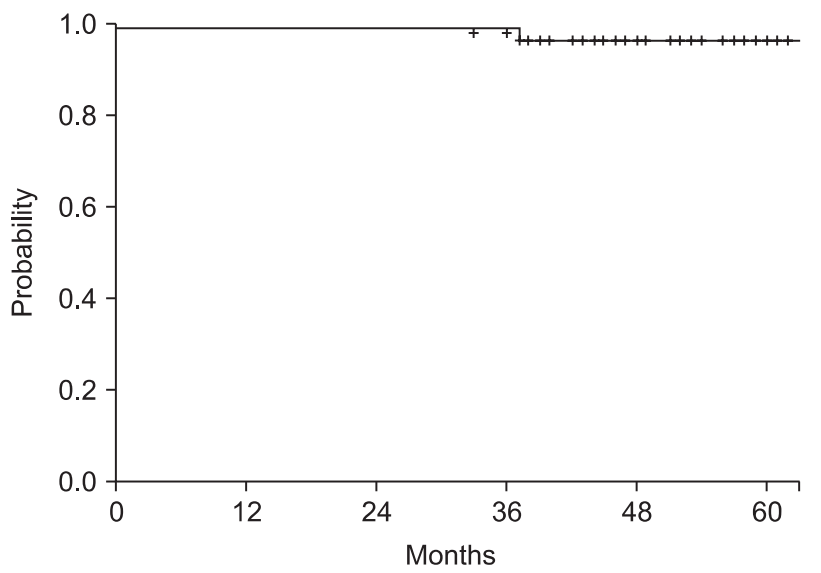

Fig. 1. (A) The 5-year biochemical relapse-free survival (bRFS) and (B) the 5 -year overall survival (OS) for all patients.

earlier. Of the remaining three, 2 received antiandrogen alone and 1 received LHRH agonist. Median period of ADT duration was 7 months (range, 1 to 30 months). Three patients were given ADT over 12 months. The distribution of patients regarding ADT timing relative to RT was as follows: $82.3 \%$ after RT, 17.6\% before RT, and 5.8\% with RT (1 patient was given both neoadjuvant and concurrent ADT), respectively.

\section{Biochemical relapse-free survival and overall survival}

Fig. 1A illustrates the 5 -year bRFS of all patients, which was estimated to be $80.4 \%$. The 5 -year OS was estimated $96.6 \%$. One patient expired due to sepsis and RT was interrupted 2 fractions before completion. The other patient died of prostate cancer progression at 37 months post-RT. The OS curve is shown in Fig. 1B.

\section{Risk factor subgroup analysis}

Each potential risk factors of biochemical relapse were subdivided into two groups. The proportion of patients and 5 -year bRFS as well as the p-value acquired in univariate analysis of each paired subgroups are shown in Table 2.

Patients with median age of 65 or younger had an estimated 5 -year bRFS of $85 \%$, which was superior to that of the older group $(78 \%, p=0.57)$, though statistically not significant. RT given in adjuvant aim yielded similar 5 -year bRFS to that of salvage aim ( $81.8 \%$ vs. $78.2 \%$, respectively; $p=0.75$ ). Intermediate or lower risk groups had better 5-year bRFS than higher risk groups ( $92.9 \%$ vs. 76.2\%, $p=0.19$ ). Patients with median pre-RT PSA $\leq 0.2 \mathrm{ng} / \mathrm{mL}$ were estimated to have 5 -year bRFS of $85.3 \%$ while median pre-RT PSA $>2 \mathrm{ng} / \mathrm{mL}$ would have $72.7 \%(p=0.24)$. Most of the adverse pathological risk factors' positivity showed lower 5-year bRFS compared to that of the corresponding negative pair.

However, the differences listed above are statistically insignificant. The two factors revealed to have significantly different 5-year bRFS after univariate analysis were ECOG and ADT use. Patients with ECOG of 0 had an improved 5-year bRFS by $22.3 \%$ points compared to ECOG $\geq 1$ ( $92.3 \%$ vs. $70.0 \%$, respectively; $p=0.03$ ) (Fig. 2). Patients who were given ADT had a 5 -year bRFS of $95.1 \%$ while the ones who were not given any had that of $40.0 \%$ ( $p<0.01$ ) (Fig. 3). However, these differences in survival did not extend to a significantly different outcome in multivariate analysis.

\section{Adverse effect}

The rate of grade $\geq 2$ acute, 3 -year actuarial chronic toxicity, and 5-year chronic toxicity are listed in Table 3. No grade 3 acute RT toxicity was observed in our cohort. Five patients (8.5\%) experienced grade 2 diarrhea requiring medication. One patient experienced grade 2 urinary incontinence and another had grade 2 erectile dysfunction.

Chronic toxicity was assessed by first analyzing the 3-year actuarial incidence of adverse effects and then evaluating the 5 -year complication rate. There was no chronic gastrointestinal complication reported at 3 years. The observed urinary toxicity at 3 years was mostly grade 1 . Five patients had mild urinary incontinence which did not necessitate safety pads. One patient had grade 2 incontinence requiring daily pad use and another patient had grade 3 incontinence which needed urinary clamp. Only 2 cases of sexual complication were observed. One patient complained of subjective erectile dysfunction but he was able to function without medication. 
Table 2. Risk factor subgroup analysis

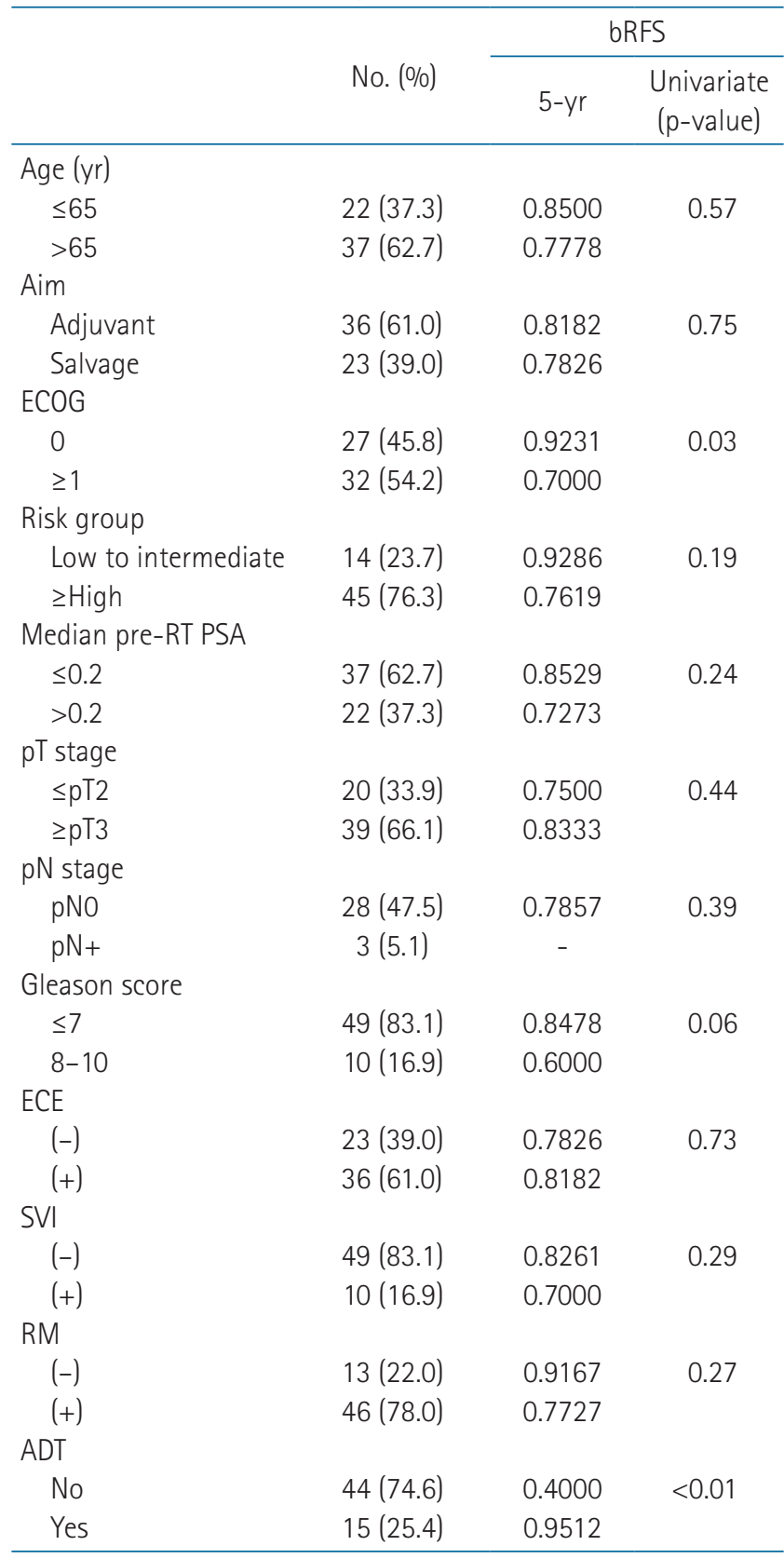

bRFS, biochemical relapse-free survival; ECOG, Eastern Cooperative Oncology Group performance status; RT, radiotherapy; PSA, prostate-specific antigen; ECE, extracapsular extension; SVI, seminal vesicle invasion; $R M$, resection margin; $A D T$, androgen deprivation therapy.

The other patient required medication.

There were 4 cases of radiation proctitis confirmed on colonoscopy at 5 years. One patient had severe diarrhea which required in-hospital care. Rectal bleeding was observed in one patient but no treatment was needed because the amount
Table 3. Grade 2 or 3 acute and chronic toxicity $(n=59)$

\begin{tabular}{lll}
\hline \multicolumn{1}{c}{ Toxicity } & Grade 2 & Grade 3 \\
\hline $\begin{array}{ll}\text { Acute toxicity } \\
\text { Gastrointestinal }\end{array}$ & $5(8.5)$ & $0(0)$ \\
Urinary & $1(1.7)$ & $0(0)$ \\
Genital & $1(1.7)$ & $0(0)$ \\
3-yr actuarial chronic toxicity & & \\
Gastrointestinal & $0(0)$ & $0(0)$ \\
Urinary & $1(1.7)$ & $1(1.7)$ \\
Genital & $1(1.7)$ & $0(0)$ \\
5-yr chronic toxicity & & \\
Gastrointestinal & $1(1.7)$ & $1(1.7)$ \\
Urinary & $3(5.1)$ & $2(3.4)$ \\
Genital & $0(0)$ & $1(1.7)$ \\
\hline
\end{tabular}

Values are presented as median (range) or number (\%).

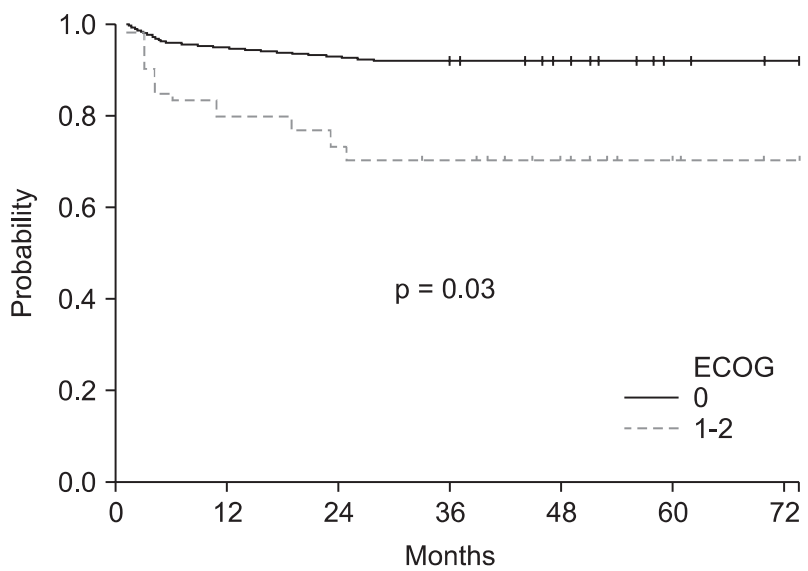

Fig. 2. The 5-year biochemical relapse-free survival difference in performance groups. ECOG, Eastern Cooperative Oncology Group.

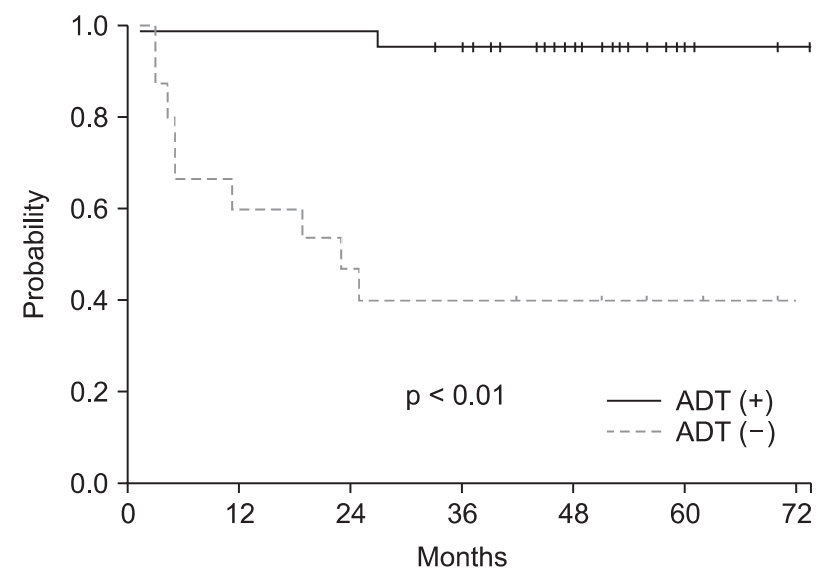

Fig. 3. The 5-year biochemical relapse-free survival difference observed by androgen deprivation therapy (ADT) use. 
Table 4. The studies on use of hormonal therapy in addition to radiation (ADT + RT) versus radiation alone

\begin{tabular}{|c|c|c|c|c|c|c|}
\hline $\begin{array}{c}\text { ADT } \\
\text { duration }\end{array}$ & ADT sequence & Study & No. & $\begin{array}{c}\text { Median } \\
\text { follow-up } \\
\text { (yr) }\end{array}$ & bRFS & p-value \\
\hline \multirow[t]{4}{*}{ Short } & $\begin{array}{l}\text { Neoadjuvant + } \\
\text { Concurrent }\end{array}$ & Eulau et al. [16] & 105 & 4.6 & $\uparrow 5-y r$ bRFS in RT + ADT (56\% vs. 27\%) & 0.0004 \\
\hline & $\begin{array}{l}\text { Neoadjuvant + } \\
\text { Concurrent }\end{array}$ & King et al. [17] & 122 & 5.9 & $\uparrow 5-y r$ bRFS in RT + ADT (57\% vs. 31\%) & 0.0012 \\
\hline & Neoadjuvant & TROG $96.01[18]$ & 818 & 10.6 & $\uparrow 10-y r$ bRFS in 3 month RT + ADT (40\% vs. 26\%) & 0.0009 \\
\hline & & & & & $\uparrow 10-y r$ bRFS in 6 month RT + ADT (47\% vs. 26\%) & 0.0001 \\
\hline \multirow[t]{2}{*}{ Long } & Adjuvant & RTOG 85-31 [19] & 141 & 6.5 & $\uparrow 5-y r$ bRFS in RT + ADT (54\% vs. 10\%) & 0.0001 \\
\hline & Adjuvant & EORTC 22863 [20] & 415 & 9.1 & $\uparrow 10-y r$ bRFS in RT + ADT (23\% vs. 48\%) & 0.0001 \\
\hline $\begin{array}{l}\text { Short vs. } \\
\text { Long }\end{array}$ & Adjuvant & RTOG 92-02 [8] & 1,554 & 11.3 & $\uparrow 10-y r$ bRFSin RT + long-term ADT (68.1\% vs. 51.9\%) & 0.0001 \\
\hline
\end{tabular}

ADT, androgen deprivation therapy; bRFS, biochemical relapse-free survival; RT, radiotherapy.

of bleeding was scanty and the symptom was intermittent. Two patients had no symptoms at all. The total number of grade $\geq 2$ urinary toxicity increased to 7 at 5 years. The erectile dysfunction treated medically at 3-year worsened and artificial inflator was inserted at 5 years.

\section{Discussion and Conclusion}

The two factors leading to significantly different 5-year actuarial bRFS in our univariate analysis were ECOG performance and androgen suppression. Performance status is a well-known prognostic indicator in various cancers, set aside prostate cancer [11].

Another factor affecting bRFS in univariate analysis was the use of hormonal therapy. Since prostate cancer in Asia is not as prevalent as in the Western part of the world, there is limitation in conducting prospective studies. Majority of the analyses dealing with ADT and postoperative RT are retrospective reviews, thus so is ours. To date, there is no completed randomized trial discussing the effect of androgen suppression in patients who received post-prostatectomy radiation. The benefit of ADT in this specific patient group is extrapolated from the established results of ADT with definitive radiation. Among the several randomized controlled trials investigating the sequence and the duration of ADT, the studies relevant to ours are summarized in Table 4.

The estimated 5 -year bRFS of our study was $80.4 \%$ for all patients after a median follow-up period of 53 months. Considering $76.3 \%$ of our patients were in the high or very high risk groups, this result is fairly comparable to previous reports. The most prominent weakness of our study is the limitation of patient number. The experiences reported by Eulau et al. [16] and King et al. [17] included more than 100 patients each, which by far outnumbers our patient group. This shortness of population size may have contributed to the outstanding bRFS of patients given both RT plus hormonal therapy. The actuarial 5-year bRFS benefit in RT + ADT group was observed with remarkable difference compared to RT alone group (95.1\% vs. 40.0\%, $p<0.01)$. This significance in univariate analysis was not sustained in multivariate analysis, however. This phenomenon may again be explained by the lack of patient number. The distribution of patients receiving multimodality therapy (44 patients) and RT alone (15 patients) were uneven, which may also have contributed to the statistical insignificance in multivariate analysis.

The results shown in Table 4 which address the benefit of ADT are in agreement with the results in our study. Majority (65\%) of patients received ADT between 6-12 months, with the median duration of 7 months. This is similar to the widely used 6-month regimen of short-term ADT. Since the decision on ADT was made by the urologists, there was heterogeneity in time which the hormonal therapy was started and the duration of the treatment caused by inter-clinician variation.

As described above, small number of patients from single institution indicates there is underlying possibility of selection bias since the sample group does not always represent the actual reality. The follow-up period was too short compared to the long survival in prostate cancer patients. The heterogeneity in radiation, hormonal therapy and patient characteristics cannot be underestimated. To overcome these intrinsic limitations and potential weaknesses commonly found in retrospective reviews such as ours, a couple of ongoing 
randomized trials have been devised to compare the effect of androgen suppression in patients receiving postoperative RT. Radiation and Androgen Deprivation in Combination after Local Surgery (RADICALS) is a phase III trial investigating the optimal timing of radiation as well as the duration ADT is given. A substudy of RADICALS randomized patients into RT alone versus 6 months or 2 years of adjuvant ADT [14]. EORTC-220433-30041 compares 64 Gy of postoperative RT alone and RT plus 6 months of androgen suppression [15]. RTOG 05-34 is investigating the role of short-term ADT in salvage setting [15]. These ongoing randomized trials are anticipated to further specify the current guidelines of treating prostate cancer.

In conclusion, the biochemical and toxicity outcome of postradical prostatectomy $\mathrm{RT}$ in our institution is comparable to those of other studies previously mentioned. A subgroup of patients given hormonal therapy had longer 5-year bRFS in univariable analysis. The role of ADT in postoperative RT cases is currently under randomized study. Until the results of these studies are reported, more inclusion of patients and further maturation of biochemical, survival and toxicity data are warranted.

\section{Conflicts of Interest}

No potential conflict of interest relevant to this article was reported.

\section{References}

1. Jung KW, Won YJ, Kong HJ, Oh CM, Seo HG, Lee JS. Cancer statistics in Korea: incidence, mortality, survival and prevalence in 2010. Cancer Res Treat 2013;45:1-14.

2. Swanson GP, Riggs $M$, Hermans M. Pathologic findings at radical prostatectomy: risk factors for failure and death. Urol Oncol 2007;25:110-4.

3. Thompson IM Jr, Tangen CM, Paradelo J, et al. Adjuvant radiotherapy for pathologically advanced prostate cancer: a randomized clinical trial. JAMA 2006;296:2329-35.

4. Wiegel T, Bottke $D$, Steiner $U$, et al. Phase III postoperative adjuvant radiotherapy after radical prostatectomy compared with radical prostatectomy alone in PT3 prostate cancer with postoperative undetectable prostate-specific antigen: ARO 96-02/AUO AP 09/95. J Clin Oncol 2009;27:2924-30.

5. Thompson IM, Tangen CM, Paradelo J, et al. Adjuvant radiotherapy for pathological T3NOMO prostate cancer significantly reduces risk of metastases and improves survival: long-term followup of a randomized clinical trial. J Urol
2009;181:956-62.

6. Bolla M, van Poppel H, Collette L, et al. Postoperative radiotherapy after radical prostatectomy: a randomised controlled trial (EORTC trial 22911). Lancet 2005;366:572-8.

7. Bolla $M$, van Poppel $H_{\text {, Tombal }}$, et al. Postoperative radiotherapy after radical prostatectomy for high-risk prostate cancer: long-term results of a randomised controlled trial (EORTC trial 22911). Lancet 2012;380:2018-27.

8. Horwitz EM, Bae K, Hanks GE, et al. Ten-year follow-up of radiation therapy oncology group protocol 92-02: a phase III trial of the duration of elective androgen deprivation in locally advanced prostate cancer. J Clin Oncol 2008;26:2497-504.

9. Pilepich MV, Winter $K_{1}$ John MJ, et al. Phase III radiation therapy oncology group (RTOG) trial 86-10 of androgen deprivation adjuvant to definitive radiotherapy in locally advanced carcinoma of the prostate. Int J Radiat Oncol Biol Phys 2001;50:1243-52.

10. Bolla M, van Tienhoven $G$, de Reijke, et al. Concomitant and adjuvant androgen deprivation (ADT) with external beam irradiation (RT) for locally advanced prostate cancer: 6 months versus 3 years ADT: results of the randomized EORTC Phase III trial 22961 [abstract]. J Clin Oncol 2007;25(18S):5014.

11. Glare P. Clinical predictors of survival in advanced cancer. J Support Oncol 2005;3:331-9.

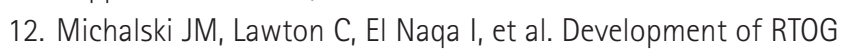
consensus guidelines for the definition of the clinical target volume for postoperative conformal radiation therapy for prostate cancer. Int J Radiat Oncol Biol Phys 2010;76:361-8.

13. Stephenson AJ, Kattan MW, Eastham JA, et al. Defining biochemical recurrence of prostate cancer after radical prostatectomy: a proposal for a standardized definition. J Clin Oncol 2006;24:3973-8.

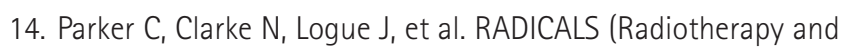
Androgen Deprivation in Combination after Local Surgery). Clin Oncol (R Coll Radiol) 2007;19:167-71.

15. Halperin EC, Wazer DE, Perez CA, Brady LW. Perez and Brady's principles and practice of radiation oncology. 6th ed. Philadelphia, PA: Wolters Kluwer/Lippincott Williams \& Wilkins; 2013.

16. Eulau SM, Tate DJ, Stamey TA, Bagshaw MA, Hancock SL. Effect of combined transient androgen deprivation and irradiation following radical prostatectomy for prostatic cancer. Int J Radiat Oncol Biol Phys 1998;41:735-40.

17. King CR, Presti JC Jr, Gill H, Brooks J, Hancock SL. Radiotherapy after radical prostatectomy: does transient androgen suppression improve outcomes? Int J Radiat Oncol Biol Phys 2004;59:341-7.

18. Denham JW, Steigler A, Lamb DS, et al. Short-term neoadjuvant androgen deprivation and radiotherapy for locally advanced prostate cancer: 10-year data from the TROG 
96.01 randomised trial. Lancet Oncol 2011;12:451-9.

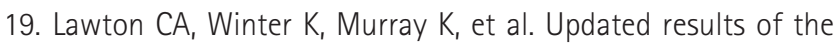
phase III Radiation Therapy Oncology Group (RTOG) trial 8531 evaluating the potential benefit of androgen suppression following standard radiation therapy for unfavorable prognosis carcinoma of the prostate. Int J Radiat Oncol Biol
Phys 2001;49:937-46.

20. Bolla M, Van Tienhoven G, Warde $P$, et al. External irradiation with or without long-term androgen suppression for prostate cancer with high metastatic risk: 10-year results of an EORTC randomised study. Lancet Oncol 2010;11:1066-73. 